\title{
Territorio y nuevas ruralidades: un recorrido teórico sobre las transformaciones de la relación campo-ciudad ${ }^{*}$
}

\author{
NAXHELLI RUIZ RIVERA** Y JAVIER DELGADO CAMPOS*** \\ ** Candidata a Doctora en Estudios del Desarrollo, University of East Anglia \\ *** Instituto de Geografía, Universidad Nacional Autónoma de México
}

\begin{abstract}
Several disciplines have discussed the formal characteristics of rural spaces, as well as the appropriate concepts for studying their reconfiguration. This essay focuses on reviewing the approaches to analyze contemporary ruralities and groups them in four categories: a) the sociologicalanalytical approaches, which refer to political, social and cultural processes that emerge as a result of globalization b) the sociological-normative approaches, which intend to understand the causes of social change in order to propose plans that generate transformation in territories and/or the rural people lifestyles c) the spatial approaches, which explore the emergence and linkages among cities of several sizes and their hinterlands and d) the neo-marxist approaches, which study the production relationships and their effects on the transformation of rural forms. The contents and theoretical assumptions of these proposals are analyzed to show the underlying different meanings of rurality and to distinguish different perspectives and methods used by each approach.
\end{abstract}

KEYWORDS: new rurality, rurbanisation, diffuse urbanization, deagrarianisation

RESUMEN En varias disciplinas se presenta una discusión sobre las características formales de los nuevos espacios rurales, y de las categorías analíticas pertinentes para estudiar su reconfiguración. En este ensayo se revisan las diversas perspectivas de estudio de la ruralidad contemporánea y los vínculos rural urbanos, agrupadas en cuatro categorías: a) enfoques sociológicos analíticos de procesos emergentes en la globalización; b) enfoques sociológicos normativos que buscan entender las causas de los cambios socioeconómicos para proponer intervenciones que impulsen esas transformaciones en los territorios rurales; c) enfoques espaciales que abordan el surgimiento y vínculos entre ciudades de distinto tamaño y su entorno; y d) enfoques neomarxistas que analizan las formas de producción y sus repercusiones en lo rural. Estas propuestas son analizadas para mostrar las formas de problematizar la(s) ruralidad(es), y diferenciar enfoques y métodos para aproximarse a ellas.

PALABRAS CLAVE: nueva ruralidad, rurbanización, urbanización difusa, desagrarización

\footnotetext{
Este trabajo es resultado del Proyecto SEMARNAT-CONACYT 01430 "Interfase rural-urbana en la Cuenca Alta del Lerma. Hacia una integración metodológica de las Ciencias Sociales y Naturales".

Recibido el 3 de noviembre de 2006, aprobado el 3 de marzo de 2008.

Correspondencia: Naxhelli Ruiz Rivera, Escuela de Estudios del Desarrollo, Universidad de East Anglia, Norwich, Norfolk, NR4 7TJ, Reino Unido. E-mail: naxhelli.ruiz@gmail.com. Javier Delgado Campos, Instituto de Geografía, Universidad Nacional Autónoma de México, Circuito Exterior, Ciudad Universitaria 04510, México, D.F. E-mail: jdelgado@igg.unam.mx.
} 


\section{Introducción}

Ante la necesidad de repensar e interpretar las formas novedosas de "lo rural", en diversas corrientes de pensamiento se ha generado una amplia discusión sobre estos espacios y las categorías analíticas pertinentes para su estudio (Ávila, 2005, p. 20).

"Nueva ruralidad", el término más aceptado, se utiliza para describir genéricamente las maneras de organización y el cambio en las funciones de los espacios tradicionalmente "no urbanos": aumento en la movilidad de personas, bienes y mensajes, deslocalización de actividades económicas, nuevos usos especializados (maquilas, segunda residencia, sitios turísticos, parques y zonas de desarrollo), surgimiento de nuevas redes sociales, así como diversificación de usos (residenciales, de esparcimiento y productivas), que los espacios rurales ejercen de manera creciente (Arias, 2002, pp. 371-377; Linck, 2001, p. 94).

Ahora bien, la acepción que se da a nueva ruralidad, varía según la disciplina de origen de quien indaga e incluye a la sociología del desarrollo, la antropología social, la geografía humana y la economía, así como intentos multidisciplinarios por combinarlas. Los ensayos que utilizan el término se pueden dividir en descriptivos y analíticos o bien en normativos de intervención, casi siempre política o productiva (Carton de Grammont, 2008, p. 26; Kay, 2008, pp. 5-6). Asimismo, el nivel de abstracción asumido varía desde la descripción de los elementos constitutivos de lo rural como hecho social, hasta una mayor elaboración conceptual de ello.

En general, asignarle tan distintas acepciones conceptuales al mismo término, debilita su capacidad explicativa. A pesar de los intentos recientes por consolidar teóricamente el concepto de nueva ruralidad, entre los que destacan los realizados por Llambí y Pérez (2007), Gómez (2008) y Ávila (2008), los marcos teóricos que se utilizan para sustentarla no confluyen en el mismo camino. Ello carga de ambigüedad a las discusiones, pues no siempre queda claro si se trata del análisis territorial de la ruralidad (y la escala involucrada), de los procesos sociales que la componen (Ramírez, 2003b, p. 53) o de las políticas de desarrollo necesarias para superar los problemas que enfrentan estas sociedades y territorios.

Cierto que aún es necesario recurrir a los indicadores convencionales de la ruralidad, tales como la densidad de población, el tamaño de la localidad, la dispersión o el peso de actividades primarias. Sin embargo, estas nociones son ya insuficientes para entender la complejidad de la ruralidad actual, que incluye temas como las dinámicas y espacios de transición territorial entre el campo y la ciudad y el estudio de las periferias urbanas. Es ya improrrogable explorar las nuevas condiciones de los espacios tradicionalmente considerados como rurales (aquellos con antecedentes económicos y culturales en actividades primarias y alejados de centros urbanos y metropolitanos) y que alojan, en forma creciente, actividades productivas secundarias y terciarias, que transforman las características y manifestaciones tradicionales de su ruralidad. Para ello, haremos un recorrido por las discusiones recientes más significativas sobre la relación campo-ciudad y los intentos por redefinirla. A partir de ello, se precisan las concepciones más recurrentes sobre las nuevas ruralidades así como el alcance y utilidad de cada una de ellas. 
El recorrido se organiza en cuatro grandes bloques temáticos. El primero de ellos, constituido por estudios de corte sociológico y cultural se centra en los actores y procesos en los que se desenvuelve la nueva ruralidad. En el segundo bloque, los estudios enfocados al desarrollo rural se caracterizan por su perfil multidisciplinario y su enfoque normativo y, además, han sugerido el enfoque de "desarrollo territorial rural" que, en cierta forma, se inscribe en la visión neoschumpeteriana sobre cambio tecnológico. Los siguientes dos grupos son más bien deductivos. El tercer bloque, de los modelos espaciales de la dinámica urbano-regional y los espacios periféricos, valiosos no sólo porque abordan explícitamente las relaciones espaciales entre la ciudad y su entorno, sino porque utilizan una terminología específica (contraurbanización, rurbanización, etc.) para referirse a los espacios periféricos, intersticiales o no urbanos, no siempre apegados a su formulación original. Por último, la cuarta sección está integrada por ensayos neomarxistas sobre la desagrarización del campo, que si bien se refieren al mismo proceso que la nueva ruralidad y otras perspectivas cercanas, lo hacen bajo un punto de vista diferente y con consecuencias políticas radicalmente distintas.

\section{Los enfoques sociológicos analíticos}

En estos enfoques prevalece el interés por visualizar las estrategias de los actores sociales ante el cambio productivo del mundo rural, en términos de los procesos políticos, sociales y culturales que enfrentan. En particular, destaca su énfasis en la fragmentación y homogeneización características de la globalización, como expresión actual de la modernidad capitalista. Una buena parte de los autores que recurren al término de nueva ruralidad o incluso el de rusticidad, se pueden incluir aquí.

Acuñado en los años noventa por la socióloga argentina Norma Giarracca (Giarracca, 1993, citada en Kay, 2008, p. 3), las primeras elaboraciones sistemáticas de nueva ruralidad se propusieron en el contexto del Consejo Latinoamericano de Ciencias Sociales (CLACSO), en Argentina y en el Instituto Interamericano de Cooperación para la Agricultura (IICA) en Costa Rica, especialmente en los trabajos compilados por Giarracca (2001) y Echeverri y Ribero (2002), aunque otros autores habían ya comenzado a explorar este ámbito anteriormente (Carton de Grammont y Tejera, 1996). A estos trabajos iniciales siguieron los significativos esfuerzos realizados por grupos de trabajo de la Pontificia Universidad Javeriana en Colombia, entre los que destacan las compilaciones de Pérez y Sumpsi (2002), Pérez y Farah (2004), Pérez, Farah y Carton de Grammont (2008). Ellos parten del supuesto de que las características que tradicionalmente han definido la ruralidad -baja densidad, predominio de actividades primarias y una vida cultural solidaria- son insuficientes para describir la situación actual de las áreas rurales en la mayor parte de América Latina, ante la desagrarización del campo y su inserción en la dinámica agroalimentaria mundial.

De acuerdo con Kay (2008, pp. 5-6), son tres las acepciones de nueva ruralidad como explicación de: i) la diversificación económica en el ámbito rural, derivada de la globalización; ii) las estrategias de gestión necesarias para alcanzar metas de desarrollo rural, tales como la competitividad económica, la sustentabilidad ambiental, la equidad de género o la reducción de la pobreza, y iii) un proyecto post-capitalista comunitario (Barkin, 2001 y 2004). En las dos primeras, en particular, se recurre a descripciones de las sociedades rurales típicas de la 
antropología, la economía y la sociología del desarrollo rural. Muchas de ellas se realizan bajo un enfoque normativo y, en ocasiones, territorial, lo que ha dado origen a una crítica sobre la ambigüedad y validez del concepto.

En el primer grupo, destaca el interés por la relación local-global que se manifiesta en los territorios y sociedades rurales, con énfasis en el papel de los actores sociales involucrados. Autores como Llambí (1996) y Long (1996) proponen entender la globalización como un "proceso socialmente construido y constantemente renegociado" (Long, 1996, p. 61) y no como algo que deba suceder fatalmente. El análisis a dos escalas (global-local) permite entender tanto los procesos generales y estructurales de la producción económica y de las instituciones como las nuevas condiciones particulares en cuanto a recursos, actores sociales, marcos de conocimiento y formas locales de organización.

Bajo esta perspectiva sociológica, el abordaje de lo local se puede resumir en las nociones de localización y relocalización. La localización es la modificación compleja de las formas locales de organización y saberes preexistentes, como resultado del cambio en las condiciones externas, mientras que la relocalización es el resurgimiento de compromisos locales y la reinvención o creación de nuevas formas sociales locales de resistencia ante las tendencias globales (Long, 1996, p. 62). Ejemplos particulares de estos cambios son los efectos de la ciencia y la tecnología en la agricultura, como la flexibilización y precarización de la fuerza de trabajo rural subordinada a formas globales de producción. En este sentido, autores como Lara y Chauvet (1996, p. 23) critican las posiciones que atribuyen a la globalización un poder homogeneizador y proponen revalorar el papel que pueden jugar los actores locales o relocalización, en términos de Long.

Otro de los conceptos asociados a la noción de nueva ruralidad centrado en la interacción de fuerzas globales y locales, es el de competitividad. De acuerdo con este enfoque, lo que diferencia a la nueva ruralidad del modelo anterior es que, si antes la lucha de los espacios rurales era por conquistar el mercado interno, ahora la producción rural trata de salir de él y orientarse al mercado externo (Lara y Chauvet, 1996, p. 32). Una aportación del enfoque de competitividad es que permite territorializar procesos sociales mediante las nociones de desigualdad y particularidad regionales, en la medida en que éstos afectan directamente la competitividad de los lugares y su capacidad de articularse a las demandas de los mercados externos.

Dentro de los enfoques sociológicos, otra propuesta que recurre a elementos de análisis similares a la nueva ruralidad, es la llamada rusticidad (Arias, 1992; Ramírez y Arias, 2002). En ésta se parte del análisis de las nuevas relaciones de producción que diversifican las actividades productivas -industriales y terciarias- en el campo, cuando se articulan con redes de producción en ciudades medias o áreas metropolitanas cercanas. Arias (2005, p. 129) resume lo más relevante de este enfoque en: a) la forma en que ciertos grupos locales enfrentan las tendencias y metamorfosis asociadas a la globalización, b) su habilidad para reelaborar y readecuar las trayectorias locales de acuerdo a las tendencias de la economía y el mercado y, c) cómo esos grupos han logrado redefinir su especialidad y rediseñar sus articulaciones espaciales. Así, la rusticidad se interesa por la invención de las nuevas modalidades de organización social mien- 
tras que la nueva ruralidad observa mejor las prácticas culturales que llevan a una apropiación particular del territorio, enfatizando los usos diferenciales de tiempo y espacio.

Desde otro ángulo analítico, Delgado (2003) plantea un sentido más restringido pero diferente de la nueva ruralidad. Para este autor, la nueva ruralidad es "el proceso político, institucional, social y cultural asociado a la rurbanización, centrado en las prácticas y estrategias de los actores en la globalización y en las nuevas localizaciones". Se trata de una definición similar a la de nueva ruralidad pero circunscrita a un ámbito espacial específico, asociándola exclusivamente con los espacios rururbanos -cuya definición veremos más adelante- mientras que en las otras acepciones el ámbito espacial del proceso no se considera como parte sustancial de su definición.

En síntesis, nueva ruralidad y rusticidad buscan explicar la diversificación de sociedades y territorios rurales. Enfatizan la dimensión socioeconómica y cultural, en cómo los actores locales se apropian de los recursos y en las dinámicas dominadas por procesos globales. Su principal mérito es haber identificado las consecuencias territoriales de la interacción global-local, dando a cada escala un peso específico. Gracias a ello, podemos afirmar que, ni las estructuras económicas y políticas globales ni los agentes locales, por sí mismos, son determinantes en la configuración de los espacios rurales, si no se atiende su vinculación específica.

\section{Los enfoques sociológicos normativos}

Bajo los enfoques normativos se estudian los nuevos procesos rurales no sólo en cuanto a su génesis y características propias como en el bloque anterior sino, además, se proponen programas y formas alternativas de intervención. Una forma de entender los cambios en las formas rurales de producción y los vínculos entre campo y ciudad, es a partir de la recuperación reciente de la vieja teoría de los ciclos económicos de Schumpeter (1982), en donde se explican los grandes cambios en la producción gracias a la adopción de innovaciones tecnológicas. Una vez maduradas, tales innovaciones tenían una influencia profunda en toda la estructura social a través de la creación de múltiples sistemas para mantener la nueva organización del trabajo.

Un ejemplo de este enfoque se puede ver en Solleiro y del Valle (1994), quienes identifican tres de estos ciclos en la producción agrícola mexicana: a) el primero constituido por el repartimiento masivo de tierras impulsada por la Reforma Agraria en los ańos treinta; b) el paradigma de la Revolución Verde - mejoramiento de semillas y métodos de cultivo-, a partir de los años cuarenta, y c) la internacionalización neoliberal, caracterizada por la penetración de las empresas transnacionales y la agroindustrialización de la producción orientada principalmente a mercados externos (Solleiro y del Valle, 1994, pp. 16-18). La transición del primero al segundo ciclo se habría caracterizado por la dominación del sector industrial sobre el agrícola, gracias a la transferencia de tecnología, lo que profundizó la polarización social existente entre diferentes productores. Posteriormente, la transición del segundo al tercer ciclo se habría debido a la pérdida de vigencia del anterior paradigma tecnológico industrial sustentado en el petróleo, y su sustitución por el predominio de los sectores electrónicos y la informática (del Valle y Lina, 1994, p. 53); de donde se desprende que la mayoría de las 
actividades rurales, agrícolas y no agrícolas participan sólo marginalmente de los mecanismos de mayor generación de valor agregado.

En esta perspectiva no es explícito el tratamiento de las mediaciones sociales y políticas que inducen o dificultan esos cambios, lo cual es mas fácil si se interroga uno sobre el papel del territorio en la innovación, la difusión de la tecnología y la competitividad.

Es el caso del Desarrollo Territorial Rural (DTR), propuesto por Alexander Schejtman y Julio Berdegué en el contexto del Centro Latinoamericano para el Desarrollo Rural (RIMISP) en Chile. El DTR es una iniciativa de investigación aplicada para impulsar "un proceso de transformación productiva e institucional en un espacio determinado", con el fin de reducir la pobreza rural (Shejtman y Berdegué, 2004, p. 4). El DTR se presenta como un proyecto de desarrollo rural, que busca articular el territorio rural a los mercados con base en la competitividad local (competitividad sistémica), junto con la institucionalidad incluyente (centrada en generar acuerdos entre actores y con ello, activos y recursos) y una visión territorial más regional, como fuente de alivio a la pobreza, con horizontes de mediano y largo plazo.

El DTR tiene como bases teóricas el llamado Desarrollo Económico Local derivado de la literatura sobre clusters y contextos competitivos junto con la literatura sobre economía institucional cercana a algunas de las teorías de generación de capital social. En términos conceptuales, el DTR se apoya en los conceptos de economías de escala, entornos de aprendizaje y gobernanza como los ejes alrededor de los cuales deben fundamentarse las estrategias de desarrollo rural.

De este posicionamiento teórico sobre el desarrollo rural y los vínculos rural-urbanos se desprenden consecuencias significativas y distintivas propias de este enfoque, que los autores resumen en siete principios. Los tres primeros se refieren a la competitividad, la difusión del conocimiento y a la necesidad de generar contextos en donde la innovación tecnológica sea una característica sustantiva del entorno. El cuarto y quinto principio se refieren a la necesidad de fortalecer los vínculos entre los territorios rurales y las ciudades (particularmente las ciudades pequeńas y medianas), ya que se considera que los territorios rurales en sí mismos no son capaces de generar los factores sustantivos para dinamizar las actividades económicas. De acuerdo con el DTR, la generación de valor de los productos rurales, que incluye tanto bienes tangibles (agrícolas, ambientales, manufacturas) como intangibles (identidad, patrimonio) está dada por la demanda urbana de dichos productos y, con ello, uno de los mecanismos de competitividad es la funcionalidad e intensidad de los vínculos urbano-rurales que puedan establecerse en un territorio dado. Finalmente, el sexto y séptimo principios se refieren a la necesidad de fortalecer la institucionalidad y la generación de capital social entre los actores asentados en el territorio, como condición para el éxito a mediano y largo plazo de los proyectos de desarrollo.

Además del DTR, existe una segunda acepción normativa de nueva ruralidad, distinta a las anteriores. En esta nueva ruralidad normativa se analizan tres factores principales asociados al territorio: la economía, la institucionalidad y los ámbitos de acción de la política (Echeverri y Ribero, 2002, pp. 18-20). Visto así, la nueva ruralidad se aplica principalmente a la gestión de 
territorios rurales, con especial interés en la revalorización del territorio y en superar la pobreza de sus habitantes, garantizar su carácter sustentable e implementar reformas institucionales y económicas (Pérez, 2001, p. 19; Pérez, 2002, p. 27). Para ello, sugieren la descentralización e integración a los mercados globales a través de la diversificación de bienes y servicios, del incremento en la productividad y rentabilidad, así como en la inversión en innovaciones tecnológicas.

En el centro de esta forma de entender la nueva ruralidad está la multifuncionalidad de los territorios rurales, en donde potencialmente pueden coexistir diversas formas de producción, comercialización y consumo de productos agrícolas y no agrícolas, un sistema de servicios ambientales y una institucionalidad reformada, basada en marcos regulatorios fuertes, junto con el fortalecimiento del capital social y de la ciudadanía entre los habitantes rurales.

En estas dos propuestas (nueva ruralidad normativa y Desarrollo Territorial Rural) se asume también una postura teórica sobre las causas del surgimiento de la nueva ruralidad aunque no profundicen en ellas. En el caso del DTR, el ámbito explicativo del cambio es su dinámica productiva y, por lo tanto, las políticas deberían orientarse al mejoramiento del ámbito productivo; las reformas institucionales o que buscan refuncionalizar los vínculos rural-urbanos, son ejemplos de ello. Esta acepción de nueva ruralidad enfatiza también la importancia de otras dimensiones, como el uso de recursos naturales o el cambio en las relaciones de género, aunque sólo DTR ha concretado programas de investigación e intervención de acuerdo con sus principios teórico-metodológicos (RIMISP, 2008).

Tras la revisión de estos dos tipos de enfoques sociológicos (analíticos y normativos), lo que se entiende por nueva ruralidad, vínculos rural-urbanos y desarrollo rural varía en contenido como en su grado de abstracción y finalidad (explicativa o normativa). En las primeras propuestas de Giarracca y Echeverri y Ribero se utilizan en sentido descriptivo, como un diagnóstico de los cambios más relevantes de la ruralidad en el contexto de la globalización. Otros autores proponen diferentes conceptos -sociológicos, económicos-, para abordar la relación globallocal y cómo los procesos sociales, en ambas escalas, determinan la llamada nueva ruralidad. La perspectiva de rusticidad se refiere también a un proceso cultural de transformación de la relación entre la organización productiva y los actores sociales, pero sin el énfasis en la globalización como los anteriores. La propuesta de Delgado abre la vertiente espacial de la nueva ruralidad, relacionándola con la rurbanización, que pone el acento en la coexistencia de ambos términos, antes que su oposición. En este primer subconjunto, la interrogación del territorio, desde el punto de vista de sus particularidades espaciales, está ausente o es muy general.

En cambio, en los enfoques normativos del segundo subconjunto, el territorio rural tiene una mayor centralidad y se considera crucial para el desarrollo. En el caso del DTR, en particular, se consideran la interacción global-local y el rol de los agentes en los procesos económicos y políticos. Sin embargo, dado que el objetivo primordial de estos enfoques es la intervención territorial -la idea de competitividad como meta económica y política- recurren más a la literatura sobre desarrollo económico regional y sobre la construcción formal e informal de la institucionalidad entre diferentes actores y grupos sociales. En este sentido, los enfoques normativos se apoyan en el vínculo rural-urbano para la obtención de sus objetivos; incluyendo 
a las ciudades dentro del área de influencia de los territorios rurales, en tanto que dinamizan y generan los procesos económicos sustantivos para una ruralidad reconstruida.

\section{Los modelos espaciales}

Los ensayos de este segundo grupo llenan un hueco importante, pero al igual que los anteriores, recurren a una diversidad de términos para referirse, básicamente, al mismo proceso.

Se puede decir que en este tipo de análisis se parte del modelo fundacional para abordar la relación espacial entre campo y ciudad: el modelo centro-periferia. Este modelo fue propuesto por H. Von Thünen en 1826, y consiste en un tipo ideal de espacio dividido en cinco anillos concéntricos alrededor de una ciudad central. En cada uno de estos anillos se alojan, preferentemente, diferentes productos primarios que demanda la población de la ciudad, considerada como su mercado "natural". Dado que la ubicación de tierra productiva es discreta y que la productividad agrícola se supera sólo mediante una intervención tecnológica, la renta de la tierra disminuye conforme aumenta la distancia al centro en función de los costos de transporte. Así, los productos más perecederos y más caros se ubican en el primer anillo, más cercano a la urbe, seguidos de los bosques, el cultivo de cereales y la cría de ganado, seguidos de una supuesta tierra "sin utilidad" (wasteland).

Sin embargo, al privilegiar el análisis de la dimensión espacial que subyace en la localización de las actividades agropecuarias con respecto a la ciudad, se descuidaron los procesos sociales claves para la comprensión de sus determinaciones. A pesar de ello, continúa vigente en el pensamiento de la relación entre el campo y la ciudad. En esta perspectiva las periferias rurales (agropecuarias) están subordinadas, en su distribución y producción, a su relación con la ciudad. La aparición de nuevos factores como las cadenas productivas, los patrones de consumo o las innovaciones tecnológicas en el transporte han transformado drásticamente el factor distancia, ampliado la escala convencional del modelo de Von Thunen y se han elaborado modelos a escala continental como el del corn belt norteamericano o europeo.

Dentro de esta perspectiva fundacional, se han elaborado recientemente otros modelos espaciales como el de la Urbanización diferencial, el de Estadios de Desarrollo Urbano, la Periurbanización y la Rurbanización. El recorrido por estos modelos es clave porque en todos ellos se reflexiona sobre la relación espacial entre campo y ciudad. De ahí ha nacido una amplia terminología, desde los términos más convencionales como conurbación, suburbanización y desconcentración, hasta los más recientes como periurbanización, rurbanización y urbanización difusa. El problema es que sus referentes empíricos y conceptuales no siempre han sido explícitos.

En el Modelo de la Urbanización Diferencial, de Geyer y Kontuly (1993), se sugiere la evolución del patrón urbano-regional de una ciudad primada y las ciudades medias y, sobre todo, pequeñas dentro de su área de influencia, a través de tres fases principales: a) Urbanización (fase de concentración); b) Polarization reversal (primera fase de la desconcentración); c) Contraurbanización (segunda fase de desconcentración). Para distinguir los umbrales de cada fase, se recurre al cambio en la migración neta que, a su vez, induce cambios en la jerarquía 
urbana. Ambos conceptos provienen del modelo de región funcional o nodal de Christaller, cuyo centro es la funcionalidad y transformaciones del sistema de ciudades.

El concepto de contraurbanización fue sugerido por primera vez para explicar la disminución de las tasas de crecimiento experimentada en las ciudades estadounidenses durante los años setenta, cuando los mayores centros urbanos perdieron población absoluta (Berry, 1976). Las primeras interpretaciones de ese cambio inesperado (en la literatura predominaba la idea de una "urbanización sin fin", como por ejemplo en la ekumenópolis de Doxiadis) lo consideraron como "una ruptura sin precedente con el pasado" (Vining y Strauss, 1977). No obstante, esa ola de descentralización no significaba el fin de la ciudad -implícito en la idea de contraurbanización-, sino que se ésta se extendía y derramaba sobre otros centros urbanos intermedios cercanos lo que, a su vez, fue interpretado como una reversión de la polarización por Richardson (1980), más que una ruptura definitiva con la ciudad principal.

Uno de los méritos del Modelo de la Urbanización Diferencial fue hacer compatibles ambas explicaciones y no considerarlas como contrapuestas como generalmente se hace. La idea de la urbanización diferenciada consiste básicamente en visualizar los cambios como un continuum urbano-urbano, a través de tres grandes periodos (ciudad principal, ciudad intermedia y ciudad pequeña, divididos en seis fases diferenciadas), que se caracterizan espacialmente por la aparición y madurez del suburbio y por procesos de descentralización hacia ciudades intermedias, primero dentro de la región y luego en regiones vecinas (Geyer y Kontuly, 1993, pp. 160-164).

Sin embargo, aunque en la última fase de la urbanización diferenciada se aprecia el crecimiento e importancia de la ciudad pequeña (small city), el modelo no se refiere a centros rurales que evolucionan hacia estadios urbanos. Es probable que muchos centros rurales lleguen a adquirir funciones urbanas de una ciudad pequeña, pero difícilmente sucede a la inversa y tampoco parece factible suponer -como muestra la realidad-, que la única perspectiva esperada de los centros rurales sea la de convertirse en ciudades. La paradoja del concepto del continuum es que acierta en la visión dinámica pero recurre a un enfoque evolucionista lineal para referirse a la situación rural actual reconocida como novedosa: o la idea del continuum es cierta y las ciudades rurales tarde o temprano se convertirán en ciudades, o habrá que buscar un concepto apropiado para caracterizar la evolución particular de estas peculiares agrociudades.

Esas nociones se pensaron desde la perspectiva de la ciudad, frente a un avance progresivo y de dominación sobre el campo, lo cual fue percibido en la ciencia regional como una difusión de la urbanización. Así, la contraurbanización, y más aun, la fase de la ciudad pequeña, expresa más una saturación del sistema urbano que el surgimiento de un ámbito (el periurbano, difuso) peculiar e inédito como resultado de la revitalización rural.

Otra variante del modelo tradicional centro-periferia es el Modelo de Estadios de Desarrollo Urbano (Berg, 1982) que busca conciliar las escalas urbana y regional involucradas en los procesos demográficos y económicos presentes en el cambio de los antiguos espacios rurales. Este modelo consta de cuatro fases: a) Urbanización, b) Suburbanización (desconcentración intrarregional y construcción de espacios periurbanos cercanos), c) Desurbanización (desconcentración 
extrarregional y construcción de espacios periurbanos lejanos) y d) Reurbanización (principalmente por gentrificación).

El modelo no se limita al mero crecimiento físico de suelo urbano o al incremento de modos de vida y comportamientos considerados como urbanos, sino que sistematiza, bajo un análisis formal, la difusión de población y empleo, en diversos momentos. Este modelo anticipa la eclosión de estudios sobre los espacios periurbanos, esos territorios genéricos que rodean a una gran ciudad, independientemente de que alojen o no, procesos de rurbanización (Delgado, 2003 p. 28). Los espacios periurbanos (llamados suburbanos o exurbia en la literatura anglosajona), se han analizado, también, a través de coronas o franjas de expansión. Para su definición, los espacios periurbanos no requieren de la condición de continuidad o conurbación y, por el contrario, implican una fragmentación o discontinuidad espacial (Ávila, 2001, p. 110).

Una interpretación histórica de los patrones periurbanos de las metrópolis industrializadas fue planteada por Jean Steinberg (1993). En su ensayo identifica tres cinturones alrededor de una ciudad: a) La periferia, o poblaciones antiguas (fauxbourgs); b) la banlieue o zonas de suelo urbano externo, y c) la zona periurbana. El primer cinturón, franja o corona, corresponde a los fauxbourgs, constituidos por poblaciones periféricas antiguas y áreas industriales de fines del siglo XIX y principios del XX. Los fauxbourgs -literalmente falsas ciudades-, eran el destino obligado para la población expulsada de la ciudad medieval o ciudad verdadera, con frecuencia, amurallada.

Tras los fauxbourgs está situado el banlieue pavillonaire (es decir, habitacional). El banlieue fue también una institución medieval para designar amplias extensiones de suelo rural bajo la jurisdicción de un landlord o propietario. En Francia, entre 1920 y 1960 fueron construidas en esta segunda corona viviendas para población de bajos ingresos. Por su parte, la zona periurbana ha sido construida sobre ambos espacios históricos, tanto fauxbourg como banlieue y se caracteriza por una compenetración entre espacios urbanos y rurales. Steinberg propuso los siguientes seis factores como las causas de este fenómeno: a) altas tasas de crecimiento poblacional, b) migración rural, c) el impacto del automóvil, d) migración intraurbana del centro a la periferia, e) la planeación de polos urbanos de crecimiento, planeados en la periferia como nuevas ciudades, centros comerciales, universidades o parques tecnológicos, y f) sitios turísticos y recreacionales.

El concepto de espacios periurbanos ha tenido particular relevancia para estudiar las áreas de transición rural-urbana ligadas a las metrópolis. La periurbanización se refiere a la emergencia y consolidación de un cinturón rural-urbano, que implica cambios en el uso de suelo tales como nueva vivienda y la relocalización de actividades económicas, y nuevas configuraciones de transportes y comunicaciones. En forma creciente, la población que vive en los poblados -con características más bien rurales en el periurbano-, trabaja en la ciudad central, a lo que Bauer y Roux (1975) llamaron rurbanización o Monclus, ciudad difusa. Aquellos usaron el término de ville eparpillé (ciudad desparramada), lo cual es irónico ya que el atributo más conspicuo de la ciudad siempre fue su supuesta concentración física y social. 
Un proceso peculiar de rurbanización ha sido identificado en Francia en espacios periurbanos con la emergencia de formas típicamente urbanas en zonas rurales cercanas a la ciudad. En esta perspectiva, el espacio rururbano es sólo uno de los tipos de espacio periurbano con características específicas, que incluyen nuevas formas de hábitat, el cambio de uso de suelo agrícola para fines urbanos y la construcción de ámbitos urbanos en áreas rurales alejadas de las zonas industriales tradicionales y de las zonas urbanas centrales.

Sin embargo, el término periurbano se aplica de formas diversas aun en Europa. Por ejemplo, en un estudio sobre el periurbano de Europa Occidental, Banzo (2005, p. 209) propone entenderlo como espacio geográfico y la periurbanización como forma de vida. Esto implica una mayor atención a la relación entre la población y el espacio, más que a la morfología o construcción del territorio mismo. Si bien se subraya que la periurbanización responde a una dinámica espacial particular, Banzo se aleja de la propuesta anterior al descartar el periurbano como un tipo de territorio. Esta opción desecha el estudio de la morfología territorial, y particularmente el problema de los límites o la forma del periurbano, al presentarlo como un falso problema. En su lugar se centra en las formas de vida resultantes de los procesos vinculados a ese espacio y, particularmente, en la gobernanza, ordenamiento y gestión ambiental de esos territorios mixtos, habitados por grupos sociales diversos. Esta perspectiva analítica -más sociológica que espacial-, la vincula con los trabajos del primer bloque que utilizan la noción de nueva ruralidad (desde su acepción normativa), aunque desde un punto de partida diferente y bajo otros supuestos teóricos.

Por lo anterior, algunos autores han utilizado, como complementarios, los términos rurbanización y nueva ruralidad: esta última está constituida por procesos que suceden sólo en los espacios de la rurbanización; todo el espacio alrededor de una ciudad es periurbano, pero sólo los que alojan nueva ruralidad, son rururbanos (Delgado, 2003, p. 15). Por eso mismo, en algunos textos franceses ambos términos se utilizan para describir procesos similares y se usan de forma indistinta (Linck, 2001, p. 91). Estos modelos se refieren a procesos que se desenvuelven en diversas escalas, entre lo urbano y lo regional, sin hacer explícito el cambio metodológico del cambio escala. La transposición de atributos de una escala a otra es la principal crítica a este tipo de enfoque (Ramírez, 2003, p. 53).

Tras este breve recorrido sobre los modelos espaciales, se observa que los dos primeros (Modelo de Urbanización Diferencial y Modelo de Estadios de Desarrollo Urbano), aunque suponen -como en Von Thunen- un espacio plano, con visibles intersticios entre las áreas urbanas que no logran caracterizar, representan un aporte sustancial para explicar el surgimiento y vínculos entre ciudades pequeñas y medianas, las dinámicas de sus áreas cercanas y de las grandes ciudades, a través del estudio de la desconcentración y los procesos migratorios. Estos modelos permiten referenciar algunas formas de relación rural-urbana en un contexto más amplio: la dinámica demográfica de los sistemas de ciudades a los que se vinculan. Sin embargo, es necesario reconocer las limitaciones que estos modelos tienen para incorporar y explicar los espacios no urbanos, los cuales representan espacios abstractos, no territorios.

Por otro lado, las nociones de rurbanización y periurbanización presentadas en esta sección cambian de objeto de estudio, aunque éste tampoco es consistente y varían desde el territorio 
como uso de suelo, hasta las dinámicas culturales y políticas que se generan e influyen en la conformación geográfica de esos contextos. Estas variaciones son una manifestación más de la necesidad de buscar explicaciones a la transición entre lo urbano y lo rural, pero también de las carencias teóricas metodológicas para definir adecuadamente las escalas, causas y características propias de estos espacios.

\section{Los enfoques neomarxistas de los nuevos procesos rurales}

El cuarto y último conjunto de análisis está centrado en el materialismo histórico y aunque se divide en tres perspectivas, con puntos de partida y argumentos distintos, concluyen de manera similar y también, como en el caso anterior, adolecen de un tratamiento propio sobre el territorio. La primera es el Vinculo Industria-Agricultura o de la subordinación excluyente; otro se refiere a la heterogeneidad de los procesos rurales en su articulación con el capital; la última es la perspectiva de la Desruralización de Wallerstein. En este apartado, más que una caracterización dura u ortodoxa del núcleo teórico de las corrientes, se pone atención a las explicaciones que privilegian el papel de las relaciones sociales de producción por encima de otros factores. En algunos de los casos, los autores no se adscriben explícitamente al marxismo, sin embargo, todos recurren a sus conceptos fundamentales.

En la teoría del Vinculo Industria-Agricultura se equipara lo rural con lo agrario y se parte de una relación entre la industria y la agricultura como una "relación de subordinación y de dominio" basada en el desarrollo desigual entre ambos sectores y cuya consecuencia más importante es la aparente desagrarización del campo (Rubio, 2001, p. 8). Esto se debe a una subordinación excluyente, noción económica que explica la marginación de la producción campesina por la agroindustrial, dentro del proceso de reproducción del capital, lo que ocasiona la exclusión de sus productores. La subordinación excluyente explica el proceso de cambio rural a través de la teoría económica marxista sobre la estructura económica agropecuaria, a diferencia de los modelos anteriores que se centran en la dimensión espacial del cambio rural.

Para otros autores de este último grupo de análisis, los intentos por revisar los conceptos sociológicos y de los modelos espaciales para explicar el cambio rural, son innecesarios (Pradilla, 2002). Este autor sostiene que la particularidad de los procesos hace imposible su generalización. Los estudios rurales, según esta perspectiva, deben hacerse a partir de estudios de caso que apliquen finalmente los instrumentos teóricos del análisis marxista existentes. Según Pradilla el análisis de los procesos actuales no requiere de nuevos conceptos, endebles, sino de un retorno a las teorías clásicas que, actualizadas ante los procesos actuales, expliquen integralmente los fenómenos de la globalización.

En esta perspectiva, las transformaciones rurales no son producto de la llamada nueva ruralidad, sino de la culminación del proceso de descampesinización ya planteado por la sociología rural marxista latinoamericana. La descampesinización es la desaparición gradual del campesino (se supone que se refiere al pequeño productor, dueño de la tierra y que no se inserta en las formas de producción agroindustriales), producto de las formas "que no funcionan en el ciclo de acumulación del capital, ahora globalizado” (Pradilla, 2002, p. 5) y del cambio tecnológico general. 
Otro ejemplo de caso de estudio bajo esta perspectiva lo ofrece De Teresa con su análisis de la producción de henequén (planta fibrosa de la familia agavácea) en Yucatán. En esta región del este mexicano, se ha cultivado el henequén históricamente, bajo distintas modalidades. Primero, la colonización española subsumió las formas prehispánicas de producción en las haciendas. Con la revolución agraria mexicana del siglo XX, se introdujo una diferencia productiva entre la forma ejidal y la privada, que sólo fue puesta en crisis por la producción de fibras sintéticas en el mercado mundial (De Teresa, 1992, pp. 71-73). Posteriormente, se introdujo un importante cambio tecnológico con la intervención del Estado para controlar los créditos, el precio de la fibra, la organización de la producción y por lo tanto, también, su productividad, todo ello a través de un organismo paraestatal creado ex-profeso (De Teresa, 1992, p. 80). En este caso no se habla propiamente de fases sino de formas de producción, en el sentido marxista, las cuales ocurren durante un periodo histórico particular.

En esta misma línea, una aportación consistente es la de Ramírez (1995; 2000; 2005) quien, sin abandonar el esquema analítico marxista, asocia las diferentes lógicas económicas de los actores involucrados en la relación campo-ciudad con la readecuación de la influencia de sus escalas geográficas. Para Ramírez, la urbanización del campo es una forma que adopta el sistema de producción rural en su redefinición ante los procesos de internacionalización de la economía. Formas, además, diferentes en cada momento histórico. Se trata del traslado, característico del postfordismo, de la industria a zonas rurales, en donde el interés conceptual por nociones como el trabajo a destajo y de organización familiar, fluctuación de los salarios e inestabilidad en el empleo, desplaza la importancia asignada en otras perspectivas, a las migraciones como factor explicativo de la relación campo/ciudad. Un esfuerzo particular que hay que abonar en su cuenta, es el de señalar la importancia de las escalas geográficas para entender el funcionamiento de las distintas lógicas discernidas.

Otro intento por explicar los cambios recientes en las zonas rurales y la relación campo-ciudad es la Desruralización de Wallerstein $(2001,2002)$. Para este autor, en el contexto actual de la economía-mundo, las dos principales estrategias del capital global para superar la crisis cíclica de largo plazo son la valorización de los recursos naturales y la urbanización del campo a través de la desruralización. Las diferencias existentes entre campo y ciudad y entre centro y periferia -dentro de la propia ciudad como entre regiones centrales y periféricas-, cumplen un papel clave en la teoría marxista -y por ello sorprende su desinterés por los modelos espaciales-, pues explican una de las principales contradicciones estructurales del capital, a saber, el del límite de la proletarización completa de la fuerza de trabajo.

La proletarización de la fuerza de trabajo y la urbanización como correlato, son condiciones necesarias para mantener la forma capitalista de acumulación, pero tiene un límite, pues eleva los costos de su reproducción. Es más costoso reproducir una fuerza de trabajo urbanizada, modernizada, sindicalizada y acostumbrada al bienestar, que una semiproletaria, en un ámbito no urbano o, más exactamente, semirrural. El segmento de la población en unidades semiproletarias (en donde el salario representa una porción mínima del ingreso), reproduce la fuerza de trabajo con menores costos, por lo que el capital puede mantener bajos salarios en el resto de los trabajadores formales. 
Ahora bien, mientras que en las áreas centrales del capital global la fuerza de trabajo modernizó y amplió su capacidad de consumo, en las periferias se reproducía en unidades domésticas semiproletarias. Aquí es donde entra la idea de la desruralización como salarización a la baja de aquella fuerza de trabajo que todavía se mantiene en actividades económicas de subsistencia, aunque sea temporalmente. Se integra a la economía monetarizada por un (bajo) ingreso salarial (sin prestaciones), a condición de que su reproducción continúe dentro de unidades domésticas (sin servicios) en áreas no urbanas, que no implican costos de urbanización y no están sujetas a normatividad ambiental o salarial o bien no se aplica. Así, la desruralización permite recuperar las tasas de ganancia y garantizar el proceso de acumulación de capital. Pero, y esa es una de las aportaciones significativas de Wallerstein, el mundo es finito y, por lo tanto, los espacios rurales por desruralizar también lo son y están principalmente en los países de la periferia.

En síntesis, en este conjunto de perspectivas neomarxistas el interés fundamental es explicar la transformación de las relaciones sociales de producción, en la cual el territorio tiene un rol muy claro para algunos autores (Wallerstein, Ramírez) y secundario para otros (Banzo, Pradilla, Rubio). Sin embargo, también dentro de esta perspectiva se han realizado aportes críticos importantes sobre la relación campo-ciudad que pueden servir como conclusión a este conjunto de propuestas. Por ejemplo, las reflexiones de Cortés (1980) retomadas por Ramírez, sobre la supuesta separación entre campo y ciudad como una falacia, ya que las esferas de la producción y consumo en ambos espacios están íntimamente relacionados: el consumo de productos rurales impulsa la capitalización del campo y la creación de circuitos de consumo rural de los productos industriales para los cuales el campo produce los insumos primarios. En ese sentido, finalmente propone que dentro del materialismo histórico no es posible concebir el campo y la ciudad como elementos opuestos sino como un sólo proceso, en el cual lo urbano y lo rural están necesariamente interrelacionados; las diferencias y desigualdades entre ambos tipos de territorio son inherentes a los mecanismos de reproducción del capital.

\section{Conclusiones}

A lo largo de este artículo hemos revisado las continuidades y diferencias entre los distintos enfoques sobre las sociedades y los territorios rurales y las propuestas de desarrollo discernidas. Los distintos enfoques se desprenden de esfuerzos interdisciplinarios, con distintos grados de éxito en cuanto a la calidad y coherencia de la síntesis teórico-metodológica que presentan, por lo cual ha sido necesario explorar y comparar los variados objetos de estudio a los que se refieren (procesos sociales, territorios o propuestas políticas).

Los trabajos de los primeros dos grupos bloques abordan los dilemas generados por los cambios en la producción, consumo y las relaciones políticas entre agentes, no sólo en las áreas rurales propiamente dichas sino también en las zonas de transición rural-urbana. Estas zonas revelan las intensas contradicciones y desigualdades sociales y territoriales entre ambos espacios. En este marco, las diferentes percepciones disciplinares del cambio social se entremezclan, generando sugerentes narrativas sobre los actores y sus relaciones a diferentes escalas pero todavía no generan un cuerpo conceptual único. En algunos casos (nueva ruralidad, DTR) llevan a propuestas de intervención para afrontar los múltiples problemas de pobreza, desigualdad, inequidad 
y degradación ambiental presentes en las sociedades y territorios rurales. Los fundamentos teóricos de este primer bloque aluden, aunque no lo expliciten, a elementos de la economía neoclásica y a los enfoques sobre la agencia de los actores sociales. Con ello, enfrentan una gran contradicción, pues “... en cierto modo, la nueva ruralidad es el resultado del neoliberalismo y promover la pluriactividad sin cambiar el contexto, es reproducir el neoliberalismo y con ello la explotación y el despojo campesino" (Kay, 2007, p. 33).

Por otro lado, los modelos espaciales sin actores explican las interacciones territoriales a través de mecanismos como la fricción de la distancia, el precio y uso del suelo, con un concepto de espacio bidimensional y un modelo de causalidad claro y simple, pero sin problematizar las causas (económicas o políticas) que influyen en los cambios territoriales.

Por su parte, los ensayos neomarxistas, uno de cuyos méritos es la exigencia crítica de coherencia teórica tienen, sin embargo, pocos mecanismos para vincular el sustrato marxista con elementos que, sin vulnerar su consistencia conceptual, enriquezcan su análisis y lo alejen del determinismo deductivo que caracteriza a algunos de sus análisis empíricos. Asimismo, con excepción de Ramírez, en la literatura latinoamericana hay pocos intentos por vincular explícitamente el análisis neomarxista de los fenómenos rurales con planteamientos de carácter espacial o territorial en donde éste no sea reducido a un mero escenario. Esto se explica, en parte, por la incompatibilidad que las diferentes aproximaciones al territorio tienen en el plano de la intervención política. Por ejemplo, la nueva ruralidad y el DTR suponen que para combatir la pobreza y la desigualdad se requieren medidas que aseguren la inserción competitiva, en los mercados globales, de las sociedades y territorios rurales. En cambio, bajo una perspectiva neomarxista, las mismas desigualdades territoriales son consecuencia de la dinámica de aquellos mercados globales, por lo que para revertir esa situación se requieren mecanismos de intervención más radicales y estructurales, dirigidos a la generación de alternativas al capitalismo y a combatir el debilitamiento de los Estados-nación, entre otras medidas.

En ese sentido, a partir de este ensayo se perfila la necesidad de profundizar el diálogo interdisciplinario, pues es claro que cada quien habla en el lenguaje especializado que conoce, bajo el tratamiento metodológico largamente aprendido, para referirse al mismo proceso y al mismo territorio. Hace falta hacer explícitos y profundizar en los vínculos teóricos y metodológicos que cada enfoque disciplinar implica, y poner de manifiesto tanto sus alcances explicativos como sus incompatibilidades internas, dado el caso. Sin esto, la diversidad de aproximaciones conceptuales y la heterogeneidad en sus características epistemológicas perderán fuerza para explicar coherentemente no sólo los vínculos rural-urbanos, sino la naturaleza de las diversas ruralidades latinoamericanas.

Otras posturas que tienen antecedentes teóricos más claros, como el DTR y el neomarxismo, requieren de un mayor reconocimiento autocrítico de sus limitaciones conceptuales y las implicaciones políticas que dichas posturas conllevan. Sin este reconocimiento, se corre el riesgo de forzar las explicaciones de los fenómenos reales a los moldes teóricos, como ya ha sucedido anteriormente. En ese sentido, es válido regresar críticamente a los cuerpos teóricos fundacionales para recuperar sus elementos explicativos más robustos y su coherencia, a con- 
dición de identificar cuándo y por qué resultan insuficientes para explicar las nuevas realidades y reconocer claramente sus potenciales consecuencias políticas.

Este trabajo pretende haber contribuido a bosquejar un mapa conceptual que oriente la tarea de comprender la diversidad de los territorios rurales y los vínculos entre diferentes sociedades y espacios. Esta exploración conceptual no es menor, ya que detrás de cada postura teórica subyace una política, con imaginarios y mecanismos de intervención propios, con importantes consecuencias para la vida presente y futura de los habitantes, ya nunca más, rurales.

\section{Referencias bibliográficas}

Arias, P. (1992). Nueva rusticidad mexicana (1 ${ }^{\text {a }}$ Ed.). México: Consejo Nacional de Ciencias y Artes.

Arias, P. (2002). Hacia el espacio rural urbano: una revisión de la relación entre el campo y la ciudad en la antropología social mexicana. Estudios Demográficos y Urbanos, 50, 363 380.

Arias, P. (2005). Nueva ruralidad: antropólogos y geógrafos frente al campo hoy. En H. Ávila, Lo urbano-rural ¿nuevas expresiones territoriales? (1a Ed., pp. 123-160). Cuernavaca: CRIM-UNAM.

Ávila, H. (2001). Ideas y planteamientos teóricos sobre los territorios periurbanos. Las relaciones campo-ciudad en algunos países de Europa y América. Investigaciones Geográficas, 45, 108-127.

Ávila, H. (2005). Introducción. Líneas de investigación y el debate en los estudios urbano-

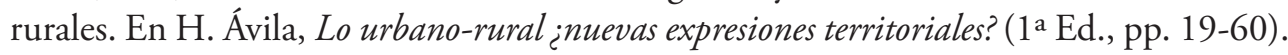
Cuernavaca: CRIM-UNAM.

Ávila, H. (2008). Enfoques geográficos en torno a la nueva ruralidad. En E. Pérez, M, Farah \& H. Carton de Grammont, La nueva ruralidad en América Latina. Avances teóricos y evidencias empíricas (1a Ed., pp. 103-132). Bogotá: Pontificia Universidad Javeriana y CLACSO.

Banzo, M. (2005). Del espacio al modo de vida. La cuestión periurbana en Europa Occidental: los casos de Francia y España. En H. Ávila, Lo urbano-rural ¿nuevas expresiones territoriales?

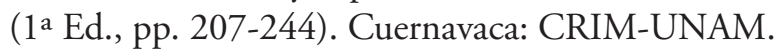

Barkin, D. (2001). Superando el paradigma neoliberal: desarrollo popular sustentable. En N. Giarracca, ¿Una nueva ruralidad en América Latina? (1a Ed., pp. 1-99). Buenos Aires: CLACSO.

Barkin, D. (2004). Who are the peasants? Latin American Research Review, 39, 3, 270-281.

Bauer, G. \& Roux, J.M. (1976). La rurbanisation ou la ville eparpillé. (1a Ed.). París: Seuil.

Berg, L. van den; Drewett, R.; Klaassen, L.; Rossi, A. \& Vijverberg, C. (1982). Urban Europe. Volume 1: a study of growth and decline (1 ${ }^{\mathrm{a}} \mathrm{Ed}$.). Oxford: Pergamon.

Berry, B. (1996). The counterurbanisation process: urban America since 1970. En H. S. Geyer $\&$ M. Kontuly (Eds.), Differential urbanization. Integrating spatial models (1 ${ }^{\text {a }}$ Ed., pp. 7-18). Londres: Arnold.

Carton de Grammont, H. \& Tejera, H. (1996). La sociedad rural mexicana frente al nuevo milenio. Tres volúmenes ( $1^{a}$ Ed.). México: INAH-UAM-UNAM-Plaza y Valdés. 
Carton de Grammont, H. (2008). El concepto de nueva ruralidad. En E. Pérez, M. Farah \& H. Carton de Grammont, La nueva ruralidad en América Latina. Avances teóricos y evidencias empiricas (1 ${ }^{\text {a }}$ Ed., pp. 23-44). Bogotá: Pontificia Universidad Javeriana y CLACSO.

Cortés, F. (1980). Conciencia teórica y metodológica: a propósito de la cuestión agraria. En I. Restrepo, Conflicto entre ciudad y campo en América Latina (1a Ed., pp. 37-51). México: Editorial Nueva Imagen.

De Teresa, A. (1992). Crisis agricola y economía campesina. El caso de los productores de henequén en Yucatán (1 ${ }^{\mathrm{a}}$ Ed.). México: UAM-Iztapalapa y Miguel Ángel Porrúa.

Del Valle, C. \& Lina, I. (1996). Modernización y rezago tecnológico en el campo y las agroindustrias. En J. Solleiro \& M. del Valle, El cambio tecnológico en la agricultura y las agroindustrias en México (1 ${ }^{a}$ Ed., pp. 51-94). México: UNAM-IIEc y Editorial Siglo XXI.

Delgado, J. (2003). Transición rural-urbana y oposición campo ciudad. En A. Aguilar, Urbanización, cambio tecnológico y costo social. El caso de la región centro de México. (1a Ed., pp. 73-118). México: Miguel Ángel Porrúa e Instituto de Geografía UNAM.

Echeverri, R. \& Ribero, M. P. (2002). Nueva ruralidad. Visión del territorio en América Latina y el Caribe. (1 $\left.1^{\text {a }} \mathrm{Ed}.\right)$. San José: IICA.

Geyer, H. \& Kontuly, T. (1993). A theoretical foundation for the concept of differential urbanization. International Regional Science Review, 15, 12, 157-177.

Giarracca, N. (1993). Los pequeños productores en la nueva ruralidad: procesos y debates. Ponencia presentada en el XIX Congreso de la Asociación Latinoamericana de Sociología, Caracas.

Giarracca, N. (2001). ¿Una nueva ruralidad en América Latina? (1a Ed.). Buenos Aires: CLACSO.

Gómez, S. (2008). Nueva ruralidad. Fundamentos teóricos y necesidad de avances empíricos. En E. Pérez, M. Farah \& H. Carton de Grammont, La nueva ruralidad en América Latina. Avances teóricos y evidencias empíricas (1ª Ed., pp. 45-78). Bogotá: Pontificia Universidad Javeriana y CLACSO.

Kay, C. (2008). Reflections on Latin American rural studies in the neoliberal globalization period: a new rurality? Documento inédito presentado en la University of East Anglia, Norwich, Reino Unido, el 16 de abril de 2008.

Lara, S. \& Chauvet, M. (1996). La inserción de la agricultura mexicana en la economía rural (1 ${ }^{a}$ Ed.). México: INAH-UAM-UNAM-Plaza y Valdés.

Linck, T. (2001). El campo en la ciudad. Reflexiones en torno a las ruralidades emergentes. Relaciones, 85, 22, 85-104.

Llambí, L. \& Pérez, E. (2007). Nuevas ruralidades y viejos campesinismos. Agenda para una nueva sociología rural latinoamericana. Cuadernos de Desarrollo Rural, 4, 59, 37-61.

Llambí, L. (1996). Globalización y nueva ruralidad en América Latina: una agenda teórica y de investigación. En S. Lara \& M. Chauvet, La inserción de la agricultura mexicana en la economía rural (1 $1^{\text {a }}$ Ed., pp. 75-98). México: INAH-UAM-UNAM-Plaza y Valdés.

Long, N. (1996). Globalización y localización: nuevos retos para la investigación rural. En S. Lara \& M. Chauvet, La inserción de la agricultura mexicana en la economía rural (1 ${ }^{\mathrm{a}} \mathrm{Ed}$. , pp. 37-74). México: INAH-UAM-UNAM-Plaza y Valdés.

Pérez, E. \& Farah, M. (2004). Desarrollo rural y nueva ruralidad en América Latina y la Unión Europea (1 ${ }^{a}$ Ed.). Bogotá: Pontificia Universidad Javeriana. 
Pérez, E. \& Sumpsi, J. (2002). Políticas, instrumentos y experiencias de desarrollo rural en América Latina y Europa (1 ${ }^{\text {a }}$ Ed.). Madrid: AECI-FODEPAL.

Pérez, E. (2001). Hacia una nueva visión de lo rural. En N. Giarraca, ¿Una nueva ruralidad en América Latina? (pp. 17-30). Buenos Aires: CLACSO. Recuperado el 24 de agosto de 2006 de www.clacso.Edu.ar

Pérez, E. (2002). Lo rural y la nueva ruralidad. En E. Pérez \& J. Sumpsi, Politicas, instrumentos

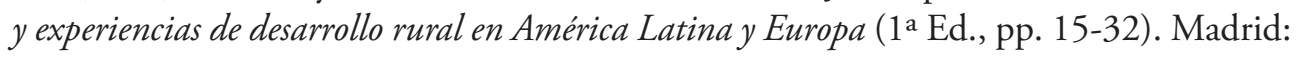
AECI-FODEPAL.

Pérez, E., Farah, M. \& Carton de Grammont, H. (2008). La nueva ruralidad en América Latina. Avances teóricos y evidencias empíricas (1 ${ }^{a}$ Ed.). Bogotá: Pontificia Universidad Javeriana y CLACSO.

Pradilla, E. (2002). Campo y ciudad en el capitalismo actual. Ciudades, 54, 3-8.

Ramírez, B. (1995). La región en su diferencia. Los valles centrales de Querétaro 1940-1990 (1a Ed.). México: UAM-Xochimilco, UAQ, Red Nacional de Investigación Urbana.

Ramírez, B. (2000). La dimensiones regionales de la relación campo-ciudad: el caso de Querétaro. En P. Torres, Procesos metropolitanos y agricultura urbana (1 ${ }^{a}$ Ed., pp. 67-86). México: UAM, Organización de las Naciones Unidas para la Agricultura y la Alimentación.

Ramírez, B. (2003). La vieja agricultura y la nueva ruralidad: enfoques y categorías desde el urbanismo y la sociología rural. Sociológica, 18, 51, 49-72.

Ramírez, B. (2005). Miradas y posturas frente a la ciudad y el campo. En H. Ávila, Lo urbanorural ¿nuevas expresiones territoriales? (1 ${ }^{\text {a }}$ Ed., pp. 61-86). Cuernavaca: CRIM-UNAM.

Ramírez, B. \& Arias, P. (2002). Hacia una nueva rusticidad. Ciudades, 54, 9-14.

Richardson, H. W. (1980). Polarization reversal in developing countries. Papers of the Regional Science Association, 45, 67-85.

RIMISP (2008). Investigación aplicada de dinámicas territoriales rurales en América Latina: marco metodológico (Versión 2). Documento de Trabajo No. 2. Santiago: Programa Dinámicas Territoriales Rurales RIMISP. Recuperado el 16 de mayo de 2008 de http:// www.rimisp.org.

Rubio, B. (2001). La exclusión de los campesinos y las nuevas corrientes teóricas de interpretación. [s.n.]

Rubio, B. (2002). De explotados a excluidos: los campesinos latinoamericanos en la fase exportadora neoliberal (1 $1^{\text {a }}$ Ed.). México: Editorial Plaza y Valdés.

Schejtman, A. \& Berdegué, J. (2004). Desarrollo territorial rural. Documento elaborado para la División América Latina y el Caribe del Fondo Internacional de Desarrollo Agrícola y el Departamento de Desarrollo Sustentable del Banco Interamericano de Desarrollo: RIMISP. Recuperado el 16 de mayo de 2008 de http://www.rimisp.org.

Schumpeter, J. (1983). The theory of economic development ( $1^{\text {a }}$ Ed. del manuscrito original de 1934). Londres: Transactions Publishers.

Solleiro, J. y Del Valle, M. (1994). El cambio tecnológico en la agricultura y las agroindustrias en México (1a Ed.). México: UNAM-IIEC y Editorial Siglo XXI.

Steinberg, J. (1993). Le periurban: definition, delimitation et specificités. Metropolisation et Periurbanisation. Cahiers du CREPIF, 42, 9-17.

Vining, D. \& Strauss, A. (1977). A demonstration that the current deconcentration of population in the UnitEd States is a clear break with the past. Environment and planning $A, 9,7,751-758$. 
Wallerstein, I. (2001). Después del liberalismo (4a Ed.). México: Siglo XXI.

Wallerstein, I. (2002). Conocer el mundo, saber el mundo. El fin de lo aprendido (2a Ed.). México: Siglo XXI. 